\title{
BALDASSAR CASTIGLIONE E GLI ISPANISMI \\ NEL CORTEGIANO (I, XXXIV). NOTE SU UN \\ EPISODIO DI AUTOCOSCIENZA LINGUISTICA, CULTURALE E POLITICA
}

Matteo Lefềre

\begin{abstract}
In this essay we analyse through a lexical, historical and pragmatic approach an important problem of Spanish and Italian Renaissance linguistics: the introduction, in Italian courtly language, of some terms derived from Castilian tradition. In fact, one of the most important carachteristics of Baldassar Castiglione's theory of language, as it is faced in the Cortegiano (1528), is its open structure that can include different kinds of words as much as terms from different countries: the only necessary condition is that all these words have to be really spoken and used by the most relevant courtiers, literates and princes. In I, xxxiv, Castiglione's deep cultural, linguistic and historical consciousness allows him to select these ispanismi in order to represent some peculiar and noble aspects of courtly way of life, as it was practiced all around the Empire: so we find some terms dealing with military life and uses as much as many others connected with art, literature and conversation.
\end{abstract}

Poco oltre la metà del primo libro del Cortegiano Baldassar Castiglione introduce il problema dei forestierismi nell'ambito della lingua cortigiana. Attraverso le discussioni degli interlocutori del trattato, sul piano linguistico emerge da parte sua l'accettazione di un criterio empirico che mostra una volontà programmatica tesa a sua volta all'omologazione di un generale principio realistico da applicarsi come regola base per la comunicazione scritta e orale - di corte. Vediamo così che Castiglione affronta il discorso sui vocaboli di origine straniera, o meglio sulla possibilità per essi di essere introdotti o meno nel lessico del gentiluomo, in due modi: da un lato, sul versante teorico, egli confida nella validità e nella disponibilità del proprio ideale linguistico eclettico; dall'altro, per ciò che riguarda l'effettiva pratica comunicativa, dà prova di una matura coscienza culturale e linguistica non solo evidenziando $\mathrm{i}$ «termini e franzesi e spagnoli» come una realtà originariamente distinta dall'idioma italiano ma anche, contemporaneamente, sottolineando il loro essere già «accettati», cioè circolanti e utilizzati, nell'orizzonte del Rinascimento. La profonda consapevolezza dell'autore di fronte al problema dei forestierismi si mostra comunque, soprattutto, nei confronti dei vocaboli di provenienza castigliana - ci riferiamo in queste pagine a quelli che egli riconosce apertamente come tali (Cortegiano, I, XXXIV) - e si esercita pertanto in più direzioni: si va dal livello meramente lessicale, con il riconoscimento della paternità spagnola per certi termini e, come vedremo, in un caso anche con la traduzione "simultanea" in italiano; a quello dell'indagine sociolinguistica, con l'allusione al campo semantico della cavalleria, che ovviamente sul piano storico-culturale si riflette naturaliter nell'universo nobiliare e quindi trova ampio spazio tra le pagine del 
Cortegiano; fino ai rilievi di natura pragmatica, con l'indicazione esplicita, come garanzia di accettabilità, del criterio dell'uso e, come discriminante, di quello della comprensione ${ }^{1}$.

Per introdurre il dibattito, va in primo luogo ricordato che nei capitoli del libro immediatamente precedenti a quello in cui viene affrontata la questione dei forestierismi l'opinione del Magnifico era stata perentoria nell'affermare che il «parlare e scriver bene» è requisito essenziale per l'uomo di corte e che «senza queste due condizioni forse tutte l'altre sariano non molto degne di laude» (Libro I, XXXI); e al parere di quest'ultimo faceva seguito il discorso del conte Ludovico, che mentre ripercorreva la storia e le caratteristiche del volgare si soffermava proprio sul problema dell'opportunità o meno dell'inserzione di alcuni termini all'interno della lingua da usarsi a corte, e che soprattutto metteva in rilievo il privilegio della sententia, cioè dell'idea da comunicare a chi legge e/o ascolta («perché il dividere le sentenzie dalle parole è un divider l'anima dal corpo», I, xxxiii). S'imponeva quindi uno dei principi essenziali della comunicazione cortigiana: la lingua è autorizzata ad inserire all'interno delle sue strutture quei vocaboli e costrutti che la rendano elegante e funzionale al discorso da veicolare, orale e scritto, grave e piacevole a seconda dei casi:

[...] bisogna dispor con bell'ordine quello che si ha a dire o scrivere; poi esprimerlo ben con le parole: le quali, s'io non m'inganno, debbono esser proprie, ellette, splendide e ben composte, ma sopra tutto usate ancor dal populo; perché quelle medesime fanno la grandezza e pompa dell'orazione, se colui che parla ha bon giudicio e diligenzia, e sa pigliar le più significative di ciò che vol dire, ed inalzarle, e come cera formandole ad arbitrio suo collocarle in tal parte $\mathrm{e}$ con tal ordine [...]. (I, XXXIII)

Di fronte alle teorie puristiche che proclamavano l'eccellenza dei modelli trecenteschi e che auspicavano nell'ambito della comunicazione letteraria un recupero della lingua di Petrarca e Boccaccio - teorie che pochi anni prima della princeps dell'opera castiglionesca avevano trovato nel Bembo delle Prose (1525) il più autorevole portabandiera - Castiglione riafferma invece la dignità di una lingua «d'uso», eclettica fino all'accoglienza di vocaboli provenienti da aree linguistiche non italiane, versatile non soltanto nell'espressione scritta ma anche e soprattutto nella comunicazione orale, da utilizzare nelle circostanze quotidiane come nelle occasioni ufficiali in nome delle esigenze più ricorrenti e dominanti in seno alla società cortigiana. Pertanto, in nome della grazia e del bon giudicio, al contrario della rigorosa "toscanità" dei modelli proposti da Bembo e dai suoi seguaci, la disputa castiglionesca approda a una visione molto più ampia del problema linguistico, autorizzando di fatto la costruzione di una koiné edificata sulle "parole splendide ed eleganti d'ogni parte d'Italia» così come, se il caso lo richiede, cioè se funzionali al

\footnotetext{
${ }^{1}$ Come vedremo, Castiglione riconduce l'utilizzo dei termini «spagnoli» proprio alle esigenze funzionali della comunicazione tra gentiluomini: "pur che sperasse esser inteso», è la condizione a cui egli vincola il "suo" cortigiano. Va poi rimarcata fin d'ora anche la profonda consapevolezza storico-culturale di Castiglione, il quale nel Libro II del trattato, mentre per bocca di Federico Fregoso indica tra le altre abilità del «perfetto cortegiano» la conoscenza delle lingue straniere, auspica soprattutto la dimestichezza proprio con lo spagnolo e con il francese, «perché il commerzio dell'una e dell'altra nazion è molto frequente in Italia e con noi sono queste due più conformi che alcuna dell'altre; e que' due prìncipi, per esser potentissimi nella guerra e splendidissimi nella pace, sempre hanno la corte piena di nobili cavalieri, che per tutto il mondo si spargono; ed a noi pur bisogna conversar con loro» (II, xxxvii). Per le citazioni dal testo castiglionesco, quando non diversamente indicato, rimandiamo sempre a B. Castiglione, Il libro del Cortegiano, introduzione di Amedeo Quondam, Milano, Garzanti, 1981.
} 
messaggio (sententia) da comunicare, su termini dall'origine più varia purché già entrati nella «consuetudine» (I, XXXIV). Nel panorama della questione della lingua così come essa si stava declinando in Italia nei primi decenni del secolo la possibilità dell'introduzione di forestierismi ormai «accettati» all'interno della sfera cortigiana rientrava proprio nella strategia castiglionesca, che si prefiggeva di scavalcare l'ortodossia bembiana in nome di una lingua «italiana, commune, copiosa e varia, e quasi come un delizioso giardino pien di diversi fiori e frutti» (I, XXXV): di fronte al monolinguismo propugnato da Bembo, Castiglione definisce un principio empirico di varietà in cui anche forestierismi e neologismi, tanto aborriti dal primo, possono collaborare a creare una lingua elegante ed armoniosa. Ma, è ovvio, le Prose focalizzano l'attenzione sul versante della retorica letteraria e dell'espressione scritta, mentre il Cortegiano riflette, empiricamente appunto, sull'orizzonte più fluido e soggetto a oscillazioni della comunicazione in senso ampio, che come abbiamo visto accoglie al suo interno tanto la dimensione dello «scrivere» quanto - e nell'universo cortigiano soprattutto - quella del «parlare»: non è un caso che nelle pagine del trattato $i$ vari personaggi si soffermino in più di una circostanza su alcuni aspetti specifici della comunicazione orale, da questioni "tecniche" quali il timbro della voce e l'accompagnamento dei gesti (I, XXXIII) alla casistica dettata dall'opportunità di certi discorsi, con i suggerimenti e i limiti in fatto di motti e facezie (II, capp. XLI e sgg.) o con le misure relative al «parlar d'amore» (III, capp. LII e sgg.).

L'indugio su un problema così specifico quale quello degli ispanismi nel Cortegiano meriterebbe in realtà più di un approfondimento in seno al dibattito linguistico del Cinquecento italiano e spagnolo; tuttavia ai fini del nostro lavoro desideriamo orientare le analisi soprattutto in direzione di alcune motivazioni di natura storico-culturale che, oltre alle ragioni strettamente linguistiche, autorizzano l'inserimento nel codice cortigiano di questi vocaboli in base a fattori di più ampia portata, e cioè, da una parte, la generale influenza della Spagna sulla cultura italiana (e viceversa!), dall'altra, la storia personale dell'autore che proprio nella penisola iberica visse gli ultimi anni della sua vita e operò la risistemazione definitiva del trattato prima della stampa veneziana del 1528 .

Alla conclusione di I, XXXIV Castiglione, proprio mentre dichiara per bocca di Ludovico di Canossa la disponibilità della lingua cortigiana ad accogliere termini provenienti da aree e tradizioni linguistiche diverse, passa dunque in rassegna quei vocaboli di origine spagnola che egli dimostra di considerare, all'altezza della stesura del testo, come già «accettati» presso le più nobili corti d'Italia:

Però a me non dispiacerebbe che, occorrendogli [al «perfetto cortegiano»], dicesse primor; dicesse accertare, avventurare; dicesse ripassare una persona con ragionamento, volendo intendere riconoscerla e trattarla per averne perfetta notizia; dicesse un cavalier senza rimprocio, attillato, creato d'un principe, ed altri tai termini, pur che sperasse esser inteso» (I, XXXIV).

Per limitarci inizialmente all'analisi storico-linguistica e lessicale di questi ispanismi, è utile soffermarsi in prima istanza sul campo semantico di riferimento e sul significato da essi assunto nei primi decenni del Cinquecento, cioè negli anni della redazione, della pubblicazione e soprattutto dell'iniziale diffusione del Cortegiano. Innanzi tutto, la coscienza linguistica castiglionesca conferma l'appartenenza di essi alla sfera della cavalleria e della nobiltà, l'aderenza a un codice assoluto d'eccellenza (etica ed estetica): tanto nella trattatistica letteraria quanto nel protocollo ufficiale e nella realtà quotidiana 
della Corte il valore e il coraggio del cavaliere («avventurar»; «senza rimprocio») così come l'alta dignità («primor») e l'eleganza dell'abito e del portamento («attillato») sono tra i requisiti principali richiesti a chiunque di quel mondo voglia far parte, sia che privilegi l'attività militare («cavalier») sia che si dedichi maggiormente a lavori d'ufficio e incarichi di servizio («creato d'un principe»). Al di là di queste annotazioni, l'accezione semantica attribuita a questi lemmi nel corso del XVI secolo permette, grazie all'attestazione in alcuni importanti lessici del tempo, di confermare sul piano del significato effettivo, storico appunto, la "perfetta" integrazione di questi ispanismi all'interno della civiltà rinascimentale e cortigiana. Se, da un lato, un'analisi di questi vocaboli in diacronia non consente di rilevare cambiamenti e oscillazioni notevoli di significato durante i vari decenni del Cinquecento, dall'altro, un esame di tali termini condotto secondo un criterio sincronico e contrastivo - con l'indagine del peculiare valore semantico assunto da ognuno di essi tanto in Italia quanto in Spagna nel periodo unitario del Rinascimento - offre spunti più interessanti, soprattutto perché permette di osservare come entrambe le nazioni nello stesso giro d'anni approdino a una definizione cosciente della «forma del vivere» cortigiana. Per ciò che riguarda il contesto spagnolo, grazie ad alcune significative imprese lessicografiche è possibile fornire uno "specchietto" sintetico dell'accezione semantica che i termini menzionati da Castiglione conobbero nella penisola iberica (ma anche, ad esempio, nei domini ispanici in Italia) durante l'intero arco del Cinquecento: ci riferiamo in particolare al Vocabulario español-latino (1495) di Antonio de Nebrija, il quale comunque operò prevalentemente tra la fine del Quattrocento e l'inizio del secolo successivo e dunque ancora nella fase embrionale del Renacimiento ${ }^{2}$; al Vocabulario spagnolo-italiano (1570) redatto da Cristóbal de las Casas, già ampiamente nella seconda metà del secolo ${ }^{3}$; e infine al Tesoro de la Lengua Castellana (1611) di Sebastián de Covarrubias, il quale compila il suo lexicon al principio del Seicento ${ }^{4}$.

\begin{tabular}{|l|l|l|l|}
\hline Castiglione & Nebrija & De las Casas & Covarrubias \\
\hline primor & $/$ & \multicolumn{1}{|c|}{$/$} & $\begin{array}{l}\text { La excelencia en el } \\
\text { arte }^{5}\end{array}$ \\
\hline accertare & $\begin{array}{l}\text { figo signum; casu } \\
\text { tango, reperio; incido }\end{array}$ & $\begin{array}{l}\text { acertar=accertare, } \\
\text { indovinare }^{7}\end{array}$ & $/$ \\
\hline
\end{tabular}

\footnotetext{
${ }^{2}$ Vocabulario español-latino, Salamanca, 1495. Citiamo però dalla successiva edizione ampliata: Dictionarium Latinohispanicum, et vice versa hispanicolatinum, Aelio Antonio Nebrissensi interprete, nunc denuo ingenti vocum accessione locupletum, pristinoque nitori sublata mendarum colluvie restitutum, Antverpiae, in aedibus Ioannis Steelsii, 1560.

${ }^{3}$ C. de las Casas, Vocabulario de las dos lenguas toscana y castellana de Christoval de las Casas..., Sevilla, Francisco Aguilar, 1570. Citiamo sempre da quest'edizione.

${ }^{4}$ S. de Covarrubias, Tesoro de la Lengua Castellana o Española, compuesto por el Licenciado Don Sebastián de Covarrubias Orozco, Capellán de Su Magestad, Mastrescuela y Canónigo de la Santa Yglesia de Cuenca, y Consultor del Santo Oficio de la Inquisición (1611), Madrid, Turner, 1977. Citiamo sempre dall'edizione moderna del testo.

${ }^{5}$ L'autore evidenzia la derivazione del termine da «Primo». Cfr. S. de Covarrubias, Tesoro..., cit., p. 882.

${ }^{6} \mathrm{Nel}$ Dictionarium latinohispanicum di Nebrija è interessante notare che «acertar» è presente in una triplice accezione: «acertar a caso, herir al blanco»= colpire, prendere a caso; «acertar en alguna cosa»= indovinare; e
} 


\begin{tabular}{|c|c|c|c|}
\hline avventurare & audeo, es, ausus sum & $\begin{array}{l}\text { aventurarse }= \\
\text { avventurarsi, arrischiarsi }{ }^{8}\end{array}$ & $*^{9}$ \\
\hline attillato & 1 & polido, ataviado $^{10}$ & 1 \\
\hline rimprocio & $\begin{array}{l}\text { reprobatio, } \\
\text { opprobrium }\end{array}$ & 1 & $\begin{array}{l}\text { Reproche = vocablo } \\
\text { antiguo, quando } \\
\text { damos en } r 1 \\
\text { ostro con alguna cosa } \\
\text { mal hecha }\end{array}$ \\
\hline ripassare... & 1 & 1 & 1 \\
\hline $\begin{array}{l}\text { creato d'un } \\
\text { principe }\end{array}$ & $\begin{array}{l}\text { alumnus; famulus, } \\
\text { mercenarius ; civilis, } \\
\text { urbanus }^{12}\end{array}$ & criado $^{13}$ & $\begin{array}{l}\text { El que sirve amo, y le } \\
\text { mantiene y da de } \\
\text { comer }^{14}\end{array}$ \\
\hline
\end{tabular}

Anche solo ad una prima analisi, appare agevole inquadrare il significato originario di questi vocaboli all'interno della lingua e soprattutto della Weltanschaaung rinascimentale: a seconda dei casi, infatti, i lessici spagnoli utilizzati consentono di omologarli al generale orizzonte semantico che delimita le abilità e le prerogative del «perfetto cortegiano». La versatilità nelle più diverse mansioni prescritta dal codice castiglionesco trova immediato

«acertar en algun lugar» = trovarsi in qualche luogo (incido, is), significato in seguito abbandonato. Cfr. A. de Nebrija, Dictionarium..., cit., p. 212v (non numerata).

${ }^{7}$ Dall'italiano la voce «accertare» rimanda a «certificar» e propriamente «acertar»; dallo spagnolo, a sua volta, il termine «acertar» rinvia a «acertare, endevenare». Cfr. C. de las Casas, Vocabulario..., cit., p. 156.

${ }^{8}$ A proposito della derivazione ispanica di questo verbo è indicativo che esso compaia come tale soltanto nella parte spagnolo-italiano, dove viene appunto riportato nella forma riflessiva «aventurarse», e non viceversa. Cfr. C. de las Casas, Vocabulario..., cit., p. 166.

${ }^{9}$ Covarrubias riporta soltanto la forma sostantivale «Aventura», che comunque riconosce come «término de libro de cavallería», aggiungendo che «llaman aventuras los acaecimientos en hechos de armas». Cfr. S. de Covarrubias, Tesoro..., cit., p. 168.

${ }^{10}$ Riportiamo il significato in castigliano poiché nella parte italiana è presente una sfumatura semantica piuttosto definita. Il primo significato, «polido», ad esempio include tra i suoi valori semantici quello di «forbito», estendendo dunque la sfera dell'eleganza dall'orizzonte del vestire, che è quello in cui prevalentemente lo inquadra Castiglione, a quello dei modi, dell'ordine e della pulizia. C. de las Casas, Vocabulario..., cit., p. 225.

${ }^{11}$ Cfr. S. de Covarrubias, Tesoro..., cit., p. 905

${ }^{12}$ In questo caso, Nebrija distingue addirittura quattro accezioni diverse: «criado que criamos» = alunno; «criado que sirve» = servitore, soldato; «criado en la ciudad» = di città; «criado en el campo»=di campagna. Quella che più si avvicina alla cultura cortigiana è chiaramente la seconda che, sia nel significato di «famulus» sia in quello di «mercenarius», contempla comunque il campo semantico del "servizio" del principe, sia che esso avvenga sul fronte domestico sia su quello militare. Cfr. A. de Nebrija, Dictionarium..., cit., p. 232r (non numerata).

${ }^{13}$ Anche in questo caso, se si conduce l'analisi in senso inverso, dallo spagnolo all'italiano, aumenta la precisione. Innanzitutto, la voce ispanica è propriamente «criado que sirve», e la serie dei rimandi declina i vari "servizi" a cui è preposto: «Famiglio, fante, [...] sbirro, sergente, valletto». Cfr. C. de las Casas, Vocabulario..., cit., p. 178.

${ }^{14}$ Come si può osservare, prevale in Covarrubias l'accezione servile del vocabolo, anche se aggiunge a questo significato generico l'espressione legata al mondo dei rapporti sociali - e che quindi si riferisce direttamente all'universo cortigiano - di «Bien criado» per alludere invece a colui «que tiene buenos respetos». Cfr. S. de Covarrubias, Tesoro..., cit., p. 370. 
riscontro nell'area semantica occupata dai differenti termini castigliani: se primor allude soprattutto alla dimensione artistica, a sua volta l'assenza di rimprocio («reproche») si raccomanda principalmente all'uomo d'armi; tutto ciò con la consapevolezza che nella società di Antico Regime proprio l'eccellenza nel mestiere delle armi e delle lettere è la condizione discriminante per la verifica della dignità e della stessa esistenza del gentiluomo di corte.

Sul fronte italiano, invece, data la patente di nobiltà linguistico-letteraria concessa a tali vocaboli di origine spagnola dall'inserzione di essi, in modo consapevole, tra le pagine del Cortegiano, è utile verificare se questi ispanismi nel corso del XVI secolo fossero o meno entrati realmente a far parte del linguaggio praticato nelle migliori corti d'Italia e proprio nell'accezione semantica proposta dal trattato. Se si consultano due opere di assoluta affidabilità quali il Vocabolario degli Accademici della $\mathrm{Crusca}^{15}$, edito all'inizio del Seicento e perciò dotato di uno sguardo retrospettivo e storicizzante rispetto al secolo precedente, e il recente e aggiornato Grande Dizionario della Lingua Italiana di S. Battaglia-G. Bàrberi Squarotti ${ }^{16}$, che riporta varie attestazioni storiche all'interno delle opere letterarie cinquecentesche, emerge un quadro piuttosto esauriente sia della penetrazione di questi termini nel repertorio linguistico e culturale italiano sia soprattutto dell'analogia, a livello di significato, con quello dell'idioma di origine:

\begin{tabular}{|l|l|}
\hline Castiglione & Italiano del ' 500 \\
\hline primor & primore=eccellenza, «dallo spagnolo primor» ${ }^{17}$ \\
\hline accertare & $\begin{array}{l}\text { testimoniare, affermare e rendere certo; indovinare; } \\
\text { cosa» }{ }^{18}\end{array}$ \\
\hline avventurare & avventurarsi, arrischiarsi ${ }^{19}$ \\
\hline attillato & ben vestito, elegante \\
\hline
\end{tabular}

${ }^{15}$ Per il Vocabolario degli Accademici della Crusca, abbiamo consultato sia la prima edizione Venezia, Giovanni Alberti, 1612 (ristampa Firenze, Licosa Reprints, 1976), sia la più ampia, Firenze, Tipografia Galileiana di M. Cellini e c., 1863 (quinta impressione), 10 voll.

${ }^{16}$ S. Battaglia-G. Bàrberi Squarotti, Grande Dizionario della Lingua Italiana, Torino, UTET, 1992, 21 voll. + 2 (Indici e Dizionario degli autori citati).

${ }^{17}$ Cfr. S. Battaglia-G. Bàrberi Squarotti, Grande Dizionario..., cit., vol. XIV, p. 367.

${ }^{18}$ Per il primo e più generale significato, cfr. Vocabolario degli Accademici della Crusca, 1612, p. 10 (citiamo sempre dalla ristampa Firenze, Licosa Reprints, 1976), che riporta attestazioni in questo senso nell'italiano a partire almeno da Dante (Commedia). Per l'ultimo significato, invece, è stato Vittorio Cian a derivare dallo spagnolo «acertar» anche la sfumatura semantica di «riuscire in una cosa, conseguire un felice successo» che, sebbene manchi di conferme nelle opere lessicografiche consultate, in effetti ben si potrebbe sposare con la "perfezione" richiesta al cortigiano. Per questa accezione e i riferimenti del caso, cfr. B. Castiglione, Il libro del Cortegiano, a cura di Vittorio Cian, Firenze, Sansoni, p. 83 n. 23.

${ }^{19} \mathrm{Da}$ «aventurar». Cfr. Vocabolario degli Accademici della Crusca, 1612, cit., p. 101.

${ }^{20}$ Dallo spagnolo «atilar», «atiladura», attestato a partire dal XVII sec. tanto per le persone («ben vestito») quanto per gli abiti («ben calzato»). La Crusca conferma poi l'applicazione del termine in riferimento all'uomo nobile e raffinato, sia esso un cavaliere o un cortigiano: «Detto di persona, vale Vestito con abiti ben adattati alla persona, ed anche con eleganza e ricercatezza», Vocabolario degli Accademici della Crusca, 1863, vol. I, p. 827. Gli 


\begin{tabular}{|l|l|}
\hline rimprocio & difetto, mancanza che suscita rimprovero ${ }^{21}$ \\
\hline $\begin{array}{l}\text { ripassare...con } \\
\text { ragionamento }\end{array}$ & sottoporre una persona a un severo interrogatorio ${ }^{22}$ \\
\hline $\begin{array}{l}\text { Creato d'un } \\
\text { principe }\end{array}$ & uomo cresciuto ed educato al servizio di un principe \\
\hline
\end{tabular}

In virtù dei riferimenti letterari che i due lessici italiani offrono copiosamente in relazione ad ognuno di questi vocaboli è quasi superfluo soffermarsi sull'uso che l'italiano cinquecentesco dovette fare di essi. Basti per il momento sottolineare come la presenza documentata di alcuni di essi, e di altri ispanismi in generale, nell'opera di un autore colto ed eclettico quale Paolo Giovio non faccia altro che confermare l'omologazione di essi nel sistema della comunicazione linguistico-letteraria del Rinascimento ${ }^{24}$ : specialmente in quegli scrittori - appunto Castiglione e Giovio, tra gli altri, in Italia, ma anche, ad esempio,

Accademici sottolineano poi che il termine compare, ad esempio, con identico significato nelle Prose di Agnolo Firenzuola, edite postume (Firenze, Lorenzo Torrentino, 1552) ma risalenti comunque agli anni del Cortegiano. Nella già utilizzata edizione veneziana del 1612, invece, per la voce «attillato» si rimandava ad «affetato» (con una sola "t"): "Quegli che usa, negli atti, nelle parole, e negli abiti soverchio artificio, e che va composto: contrario a sciatto, e a sciamannato», Vocabolario degli Accademici della Crusca, 1612, p. 25.

${ }^{21} \mathrm{Da}$ «reproche». Il Battaglia riporta più che altro "rimpròccio", con tutte le sue varianti «(ant. rempròchio, rimbòccio, rimbròccio, rimpròcchio, rimpròcio)», e tra gli esempi sottolinea proprio la frase di Castiglione per alludere a «difetto, fallo, mancanza che provoca o suscita rimprovero e biasimo», S. Battaglia-G. Bàrberi Squarotti, Grande Dizionario..., cit., vol. XVI, p. 476; ma soprattutto, per le attestazioni precedenti, rimanda anche a Guittone d'Arezzo, che lo usa nel senso di «Azione, comportamento o situazione che costituisce un forte rimprovero o un motivo di vergogna, come confronto e paragone, per qualcuno», Ibid. Infine, si segnala anche la forma "ripròccio", con lo stesso significato, di cui è documentata la presenza in Paolo Giovio. Cfr. ivi, p. 717.

${ }^{22}$ Cfr. S. Battaglia-G. Bàrberi Squarotti, Grande Dizionario..., cit., vol. XVI, p. 644. Battaglia cita, tra gli esempi cinquecenteschi, ancora le parole di Castiglione, di cui l'autore stesso offre al lettore una "traduzione simultanea" e una resa efficace: «riconoscerla e trattarla per averne perfetta notizia».

${ }^{23} \mathrm{Da}$ «criado». Anche in questo caso si conferma esplicitamente l'origine e l'accezione spagnola del termine: «Dicevasi un tempo Colui che era stato creato, ossia educato, nella casa di qualche Grande, o anche di privato cittadino, dal quale poi era investito di alcun grado o ufficio, ovvero tirato su in una data professione o arte, o comecchessia bene avviato, onde spesso diveniva suo dipendente. Dallo spagn. criado», Vocabolario degli Accademici della Crusca, 1863, vol. III, p. 958, con varie attestazioni cinquecentesche.

${ }^{24}$ In generale, ispanismi e forestierismi risultano per lo più evitati dai puristi della lingua, dai "bembiani", mentre si trovano invece nei fautori delle tesi realistiche che stabiliscono un principio eclettico per la definizione e lo sviluppo di una lingua cortigiana: oltre a Castiglione, ad esempio, anche le Lettere di Paolo Giovio e il suo Dialogo delle imprese danno ormai come acquisiti diversi ispanismi. Tuttavia, come ha messo in evidenza Gian Luigi Beccaria, anche tra i principali e più autorevoli promotori dell'eclettismo linguistico cortigiano molte volte l'autore non cede al forestierismo per il timore che esso possa essere percepito come episodio di "affettazione": «Ad esempio, la posizione di uno scrittore aperto come il Castiglione al forestierismo, ma schivo dall'«affettazione», spiega perché lo spagnolo sosiego sia reso con il calco riposo, gravità riposata, e non con sussiego; spiega lo scrupolo di evitare l'affettato forestierismo sosegato, presente nelle prime redazioni del Cortegiano, ma che non arriva alla redazione definitiva». G. L. Beccaria, Riflessi linguistici dello spagnolo sull italiano del primo cinquecento, in AA.VV, Doce consideraciones sobre el mundo hispano-italiano en tiempos de Alfonso I y Juan de Valdés. Actas del Coloquio interdisciplinar (Bologna, aprile 1976), Roma, Instituto Español de Lengua y Literatura de Roma, 1979, p. 105. 
Juan de Valdés per il contesto spagnolo ${ }^{25}$ - in cui più forte fu la scelta consapevole in direzione di una lingua che fondasse le proprie ragioni costitutive sul criterio realistico dell'uso, si offrì piena cittadinanza a tutti quei lemmi che, indipendentemente dalla "purezza" originaria, fossero ormai «accettati» in seno alla nobile élite cortigiana tanto italiana quanto spagnola.

Alla luce di queste brevi considerazioni e in seguito all'indagine condotta su questi vocaboli è dunque possibile tracciare un quadro sintetico del rapporto tra $\mathrm{i}$ due volgari, italiano e castigliano, di fronte all'urgenza della definizione e della codificazione di una lingua cortigiana. Così, se si riuniscono insieme i dati riportati nelle due tabelle precedenti, con le dovute eccezioni e con le annotazioni del caso, la stretta relazione e l'omogeneità lessicale e semantica tra i due idiomi appare piuttosto evidente:

\begin{tabular}{|c|c|c|c|c|}
\hline Castiglione & Italiano del '500 & Nebrija & De las Casas & Covarrubias \\
\hline primor & eccellenza & 1 & 1 & $\begin{array}{l}\text { La excelencia en } \\
\text { el arte }\end{array}$ \\
\hline accertare & $\begin{array}{l}\text { testimoniare, } \\
\text { accertare; } \\
\text { indovinare; } \\
\text { riuscire in una } \\
\text { cosa }\end{array}$ & $\begin{array}{l}\text { figo } \quad \text { signum; } \\
\text { casu } \quad \text { tango, } \\
\text { reperio; incido }\end{array}$ & $\begin{array}{l}\text { acertar }=\text { accertare, } \\
\text { indovinare }\end{array}$ & 1 \\
\hline avventurare & $\begin{array}{l}\text { Avventurarsi, } \\
\text { arrischiarsi }\end{array}$ & audeo, es, ausus & $\begin{array}{l}\text { aventurarse }= \\
\text { avventurarsi, } \\
\text { arrischiarsi }\end{array}$ & * \\
\hline attillato & $\begin{array}{l}\text { ben vestito, } \\
\text { elegante }\end{array}$ & 1 & polido, ataviado & 1 \\
\hline rimprocio & $\begin{array}{l}\text { difetto, } \\
\text { mancanza }\end{array}$ & $\begin{array}{l}\text { reprobatio, } \\
\text { opprobrium }\end{array}$ & l & $\begin{array}{l}\text { Vocablo antiguo, } \\
\text { quando damos en } \\
\text { rostro con alguna } \\
\text { cosa mal hecha }\end{array}$ \\
\hline $\begin{array}{l}\text { ripassare... } \\
\text { con } \\
\text { ragionamento } \\
\end{array}$ & $\begin{array}{l}\text { sottoporre una } \\
\text { persona a } \\
\text { interrogatorio }\end{array}$ & 1 & 1 & 1 \\
\hline $\begin{array}{l}\text { creato d'un } \\
\text { principe }\end{array}$ & $\begin{array}{l}\text { uomo educato al } \\
\text { servizio di un } \\
\text { principe }\end{array}$ & $\begin{array}{l}\text { alumnus; } \\
\text { famulus, } \\
\text { mercenarius ; } \\
\text { civilis, urbanus }\end{array}$ & criado & $\begin{array}{l}\text { El que sirve amo, } \\
\text { y le mantiene y } \\
\text { da de comer }\end{array}$ \\
\hline
\end{tabular}

\footnotetext{
${ }^{25}$ Per un primo sguardo sulle consonanze in fatto di teoria linguistica tra Valdés e Castiglione, cfr. soprattutto L. Terracini, Lingua come problema nella letteratura spagnola del Cinquecento (con una frangia cervantina), Torino, Stampatori, 1979, in part. pp. 18-21; ma anche M. Bataillon, Erasmo y España, México, Fondo de cultura económica, 1950, vol. II, p. 310; e R. Lapesa, Introducción a J. de Valdés, Diálogo de la lengua, edición de R. Lapesa, Zaragoza, Ebro, 1940, in part. p. 21.
} 
Senza indugiare sulle minime sfumature di significato che pure emergono qua e là dal confronto di lingue, l'affermazione castiglionesca in fatto di "accettabilità" di questi termini appare pertanto fuori discussione, dal momento che la lingua cortigiana non solo immette per "consuetudine" ed eleganza - questi ispanismi all'interno del proprio repertorio lessicale, ma per lo più rispetta e fa propri i valori culturali e ideologici da essi in origine contemplati. Se, come abbiamo visto, nel Vocabulario español-latino di Nebrija - nel caso di «acertar», ad esempio - sussiste per ovvie ragioni costitutive l'accezione latineggiante di «trovarsi» (da incido, is), nei lessici successivi, redatti tra la seconda metà del Cinquecento (De las Casas) e il primo Seicento (Covarrubias), i vocaboli citati da Castiglione appaiono inseriti "perfettamente" nell'universo cortigiano; anzi, probabilmente è proprio nella dimensione della cortesanía che trovano il loro significato più vivo e durevole, giungendo con tutto il portato culturale da essi riassunto fino al secolo successivo. In pratica, all'interno della koiné castiglionesca non esistono pregiudizi puristici o nazionalistici e perciò a livello di comunicazione di corte, non si percepiscono fratture o spostamenti tra il lessico italiano e quello castigliano ${ }^{26}$. Per Castiglione la «regula universalissima» incarnata nella triade «grazia», «sprezzatura», «bon giudicio»-governa un intero codice di comportamento di cui la norma linguistica rappresenta solo una parte; e così dato che $\mathrm{i}$ princìi del «parlare e scriver bene» devono amalgamarsi in modo armonico con le altre qualità del «perfetto cortigiano», all'autore non interessa tanto la rigorosità in senso puristico della lingua quanto piuttosto la disponibilità di essa ad accogliere al suo interno, in modo eclettico, vocaboli di diversa provenienza purché anche questi collaborino a rendere operativa una "forma del comunicare" tanto nobile quanto l'emittente (cortigiano) e i destinatari (principe/cortigiani) del messaggio, nonché il suo referente, cioè l'orizzonte della Corte. Il realismo linguistico castiglionesco si mostra dunque aperto alle voci più disparate in nome della funzionalità degli strumenti della comunicazione e soprattutto della dignità dell'eloquio e della «sentenzia», in ovvia analogia con il contesto d'eccellenza in cui operano gli interlocutori del trattato: parliamo di un orizzonte linguistico, ideologico, sociale e culturale - la Corte - all'interno del quale soltanto è concepibile una lingua «copiosa e varia» e opportunamente paragonata - lo ripetiamo - a un «delizioso giardino», alla stregua di uno dei tanti che certamente circondavano il palazzo dei Duchi d'Urbino o la residenza di Carlo V a Toledo.

Al di là dei rilievi lessicali, comunque, desideriamo qui approfondire anche le ragioni specifiche per cui Castiglione si sofferma, sia pure per poche righe, proprio su vocaboli di provenienza spagnola. In questo senso, la vicenda umana e professionale dell'autore nonché la situazione politica internazionale, e italiana in particolare, dei primi decenni del Cinquecento offrono più di uno spunto per comprendere appieno il valore culturale ancor prima che linguistico delle scelte castiglionesche.

Baldassar Castiglione, com'è noto, trascorse gli ultimi anni della sua vita - che coincisero altresì con il periodo dell'ultima revisione del Cortegiano - proprio in Spagna: conobbe infatti il momento più alto della sua carriera diplomatica quando prestò servizio

\footnotetext{
${ }^{26}$ Boscán, ad esempio, nella sua versione castigliana del trattato non sente neppure la necessità di riportare tutti i termini menzionati da Castiglione nella versione originale e si limita a citare «acertar» e «aventurar». Cfr. Baltasar de Castiglione, El Cortesano, introducción y notas de Rogelio Reyes Cano, quinta edición revisada y ampliada, Madrid, Espasa-Calpe, 1984, p. 113.
} 
alla corte di Carlo V come nunzio apostolico di papa Clemente VII. A Toledo, dove era giunto 1'11 marzo del 1525, Castiglione dovette affrontare una situazione politica internazionale piuttosto complicata e si trovò quasi sempre in difficoltà tra le indecisioni tendenziose e le «tresche antispagnole» di Sua Santità, da una parte, e la propria professionalità e le proprie convinzioni, dall'altra' ${ }^{27}$. L'esito della battaglia di Pavia, combattuta nello stesso anno della sua partenza alla volta della penisola iberica, con la vittoria spagnola e la cattura di Francesco I, aveva ulteriormente spostato i già sbilanciati equilibri europei dalla parte di Carlo, e la successiva Pace di Madrid (gennaio 1526) si sarebbe presto rivelata una semplice e breve interruzione rispetto a un conflitto le cui soluzioni non erano ancora minimamente ipotizzabili. Comunque, a complicare il lavoro di Castiglione presso l'Imperatore, e quasi per dissipare gli ultimi dubbi di quest'ultimo sulla malizia filofrancese e l'incostanza di Clemente VII, il pontefice in persona di lì a pochi mesi stipulò la Lega di Cognac, che alla fine dei conti non fece altro che eliminare gli scrupoli dell'Asburgo nell'ordinare il Sacco di Roma del maggio del '27. Inoltre, risale a questi anni di turbolenza per la storia d'Europa anche l'amicizia tra Castiglione e Boscán, il primo traduttore spagnolo (ed europeo) del Cortegiano, che con la sua versione castigliana del trattato cambiò radicalmente la storia delle lettere e delle pratiche sociali della Spagna di Antico Regime ${ }^{28}$.

Castiglione ebbe dunque con la realtà culturale e linguistica spagnola uno stretto legame tanto per le urgenze professionali quanto per la propria vicenda di uomo e di letterato, viste le sue illustri amicizie con i migliori umanisti iberici del tempo e soprattutto visto che nella penisola iberica profuse l'impegno di revisione del Cortegiano prima dell'invio del manoscritto da Toledo a Venezia per la stampa. Gli anni trascorsi alla corte imperiale gli permisero altresì di acquisire una certa padronanza della lingua castigliana e una

\footnotetext{
${ }^{27}$ Come ha sottolineato Vittorio Cian, «[... da un lato, stava il suo primo dovere, quello d'interpretare i voleri del Papa, la cui politica oscillante era un continuo zig zag; dall'altro, la sua fede ben nota, d'imperiale convinto, sempre animosamente professata nella stessa e per la stessa Curia di Roma; una fede maturatasi alla stregua d'una non breve esperienza e fondata sulla persuasione di una reale superiorità della potenza spagnola, destinata a ricevere la solenne conferma nel Parco della Certosa pavese», V. Cian, Un illustre nunzio pontificio: Baldassar Castiglione, Città del Vaticano, Biblioteca Apostolica Vaticana, 1951, p. 112. Comunque, per tutto ciò che riguarda la permanenza di Castiglione in Spagna e con una grandissima abbondanza di dati, cfr. anche M. Menéndez Pelayo, Boscán y sus obras poéticas, cit., to. XIII, pp. 79 e sgg.; ma si vedano, tra gli altri, anche G. Martinati, Castiglione, nunzio apostolico alla corte di Spagna. Notizie storico-biografiche intorno al Conte Baldassarre Castiglione, Firenze, s. n., 1890; C. Dionisotti, Recensione a Vittorio Cian, Un illustre nunzio pontificio del Rinascimento: Baldassar Castiglione, in "Giornale storico della letteratura italiana», 1952, pp. 3157; J. Guidi, L'Espagne dans la vie et dans l'oeuvre de B. Castiglione: de l'équilibre franco-hispanique au choix impérial, in AA. VV., Présence et influence de l'Espagne dans la culture italienne de la Renaissance, Centre de recherche sur la Renaissance italienne, Paris, Université de la Sorbonne, 1978, pp. 113-202; e, dello stesso autore, Baldassar Castiglione et le pouvoir politique: du gentilhomme de cour au nonce pontifical, in Les écrivains et le pouvoir en Italie à l'époque de la Renaissance, Paris, Université de la Sorbonne Nouvelle, 1973, pp. 243-278.

${ }^{28}$ Non è qui possibile elencare tutti i saggi che hanno evidenziato l'importanza del Libro del Cortegiano nel contesto internazionale di Antico Regime. Rimandiamo pertanto in questa sede ad alcuni studi recenti: P. Burke, The fortunes of the "Courtier", Cambridge, Polity Press, 1995, trad.it. Le fortune del "Cortegiano". Baldassarre Castiglione e $i$ percorsi del Rinascimento europeo, Roma, Donzelli, 1998; il secondo volume dell'ampia riflessione di C. Ossola-A. Prosperi, La Corte e il "Cortegiano", I: La scena del testo, a cura di Carlo Ossola; II: Un modello europeo, a cura di Adriano Prosperi, Roma, Bulzoni, 1980; e ovviamente lo studio complessivo di A. Quondam, "Questo povero cortegiano». Castiglione, il Libro, la Storia, Roma, Bulzoni, 2000, che dedica molte riflessioni all'analisi della dimensione europea dell'opera, soprattutto inquadrandola nel contesto geografico, storico e culturale della realtà sovranazionale dell'impero di Carlo V.
} 
dimestichezza con le «cose di Spagna» che si riflette non solo in alcuni luoghi del trattato ma anche nelle missive di natura ufficiale o privata. Ad esempio, nella lettera con cui il nunzio pontificio esprimeva al segretario imperiale Alfonso de Valdés tutta la sua costernazione per i tristi eventi del Sacco, e quindi in una circostanza tutt'altro che distesa, Castiglione si riferiva alla Spagna con parole che al di là della cautela diplomatica dimostravano comunque ammirazione e gratitudine verso la nazione che ormai da vari anni lo ospitava: «E bench'io abbia ricevuto tanto onore e tante cortesie da questa eccellentissima nazione, che mai non sono per scordarmele, tal ch'io non mi riputarò giamai di essere meno spagnuolo che italiano $[\ldots] \gg .{ }^{29}$ Certo, nel prosieguo della sua risposta al Segretario, e soprattutto nei suoi rapporti con lui, i toni si sarebbero fatti sempre meno concilianti, tuttavia la autodichiarazione di «spagnolità», al di là della pratica della prudenza fa riflettere sul rispetto che Castigkione dovette avere nei confronti della realtà spagnola e che trova conferma alla luce dei già più volte ricordati accenni al costume e alla lingua iberica presenti nelle pagine del Cortegiano.

Come ha mostrato G. L. Beccaria, per Castiglione si può parlare di «bilinguismo individuale» ${ }^{30}$, dal momento che la professione di diplomatico richiedeva la conoscenza di quella lingua castigliana che dopo decenni di propaganda - sulla scia della prospettiva universalistica tracciata a fine Quattrocento da Antonio de Nebrija - risultava essere realmente la lingua dell'Impero, cioè l'idioma più diffuso in Europa in virtù dei successi politici di Carlo V e soprattutto grazie alle vittorie del suo esercito internazionale. E Castiglione, nel momento in cui alludeva alla possibilità di introdurre certi vocaboli spagnoli nel lessico cortigiano, aveva senza dubbio presente la propria consuetudine linguistica nei confronti di un idioma la cui «gravità» e «piacevolezza» conosceva ormai da anni. Lo stesso ideale eclettico proposto per la definizione di una lingua della comunicazione cortigiana nasce, del resto, non solo da una personale presa di posizione, o da un'algida scelta di metodo, ma si fonda sulla nozione dell'esperienza, che a Castiglione derivava dalla conoscenza diretta degli usi linguistici delle migliori corti italiane ed europee - visto il suo lavoro, era quotidianamente a contatto con la diplomazia di mezza Europa - e soprattutto dalla naturale o obbligata dimestichezza con più idiomi - latino, italiano, spagnolo, francese - che appunto la sua formazione culturale, la sua vicenda umana e le necessità professionali gli richiedevano. In ogni caso, sul fronte della generale penetrazione degli ispanismi nella lingua e nella cultura italiana del primo Cinquecento, l'ambito più intensamente interessato è senz'altro proprio quello che riconduce all'aspetto mondano del vivere sociale: in questi anni si assiste a un proliferare di termini di provenienza iberica che si riferiscono al gioco (carte, giostre, tornei ecc.), al cavallo, alla danza, al vestire, e all'interno del mondo cortigiano, comunque, tanto in Castiglione quanto in molta altra letteratura dei primi decenni del Cinquecento, attraverso l'elemento linguistico si può osservare un'autentica consonanza tra principi culturali e ideologici: è il caso del celebre

\footnotetext{
${ }^{29}$ B. Castiglione, Lettere, «Risposta al Valdés», in Il libro del Cortegiano con una scelta delle opere minori, a cura di Bruno Maier, Torino, UTET, 1964 (II ed.), p. 693, citato anche in F. Fernández Murga, El saco de Roma en los escritores italianos y españoles de la época, in AA.VV., Doce consideraciones..., cit., p. 51. Anche Lore Terracini ha ribadito che $«$ Gli italiani $[. .$.$] non potevano negare una certa affinità tra loro e gli spagnoli, come fa Castiglione,$ il quale appunto è pronto ad aprire le porte dell'italiano a voci castigliane quando la materia lo renda necessario». L. Terracini, Lingua come problema.., cit., pp. 11-12.

${ }^{30}$ Cfr. G. L. Beccaria, Riflessi linguistici dello spagnolo..., cit., pp. 103-114 (in part. p. 105).
} 
ispanismo «disinvoltura» - comportamento naturale e insieme elegante, misurato e armonico, in nome della «sprezzatura» - che appare il riflesso di una società che anche in un solo termine consacra alcuni dei propri ideali estetici e sociali. Il neologismo - dallo spagnolo «desenvoltura», in questo caso - attecchisce, così come altri ispanismi organici all'orizzonte cortigiano (creanza, galante, sfarzo, brio ecc.), poiché il contesto di ricezione è pronto ad accoglierlo e declinarlo sul piano semantico in virtù di un'ideologia e di una cultura comuni, in questo caso la dimensione sociale cortigiana: si tratta allora di una profonda relazione tra due lingue in quanto espressioni di una serie di valori in cui una civiltà tutta si riconosce, e non dell'acquisizione di elementi puramente stilistici (gusto per l'esotico, effetto di ridondanza, ornamento lessicale ecc.) $)^{31}$.

Infine, la diffusione degli ispanismi linguistici nella letteratura rinascimentale non è comunque dettata esclusivamente da ragioni di affinità a livello di gusti e stile tra $i$ gentiluomini delle due nazioni o dalla partecipazione ai medesimi riti della mondanità. La presenza della Spagna in Italia durante il XVI secolo è infatti una costante non soltanto della lingua e della letteratura di corte ma anche e soprattutto, come accennato, della più ampia dimensione politica e sociale: a seguito delle vittorie degli eserciti dei Re Cattolici, prima, e di Carlo V, poi, durante le Guerre d'Italia si origina dalla penisola iberica un viavai costante di dignitari, comandanti, soldati e segretari che affiancano i rappresentanti del potere imperiale nello svolgimento dei lavori di amministrazione, segreteria e ordine pubblico nei territori progressivamente occupati. Pertanto l'elemento ispanico, che pure trova piena cittadinanza nel mondo della comunicazione cortigiana, si diffonde altresì a livello di uso quotidiano nelle cancellerie dei funzionari imperiali, nei quartieri riservati alle truppe di stanza in Italia e in tutte le strade delle città toccate dalla conquista spagnola. La lingua castigliana, con il passare dei decenni del Cinquecento e in modo direttamente proporzionale all'espansione territoriale e politica dell'Impero, acquisisce dunque un'importanza assoluta sul piano europeo, e Castiglione, mettendo in bocca al "suo" cortigiano quei termini spagnoli ormai «accettati» nella realtà linguistica italiana, mostra anche sul piano del lessico quell'intelligenza e quel realismo che in più di una occasione lo avevano accompagnato nel corso della sua attività diplomatica. Con buona pace dei puristi e degli arcaisti, all'interno del dibattito sulla questione della lingua egli opta per una visione aperta, moderna e soprattutto aggiornata rispetto all'andamento delle vicende non solo intellettuali ma anche politiche dell'Europa del Cinquecento: dinanzi al rischio di rinchiudere la lingua e la sua dinamicità dentro i confini angusti delle opzioni puristiche, un uomo esperto delle «cose» della corte e del mondo preferisce mantenere una posizione eclettica e relativista, al fine di offrire all'idioma una maggiore disponibilità comunicativa orale e scritta - nei confronti dei più diversi volgari, in primo luogo quella «castellana lengua» che si diffondeva in modo sempre più rapido e prepotente su tutto lo scacchiere internazionale ${ }^{32}$. Sebbene gli ispanismi "dichiarati" del Cortegiano, cioè quelli di cui

\footnotetext{
${ }^{31}$ Per tutto questo discorso, cfr. sempre ivi, in particolare pp. 111-112.

${ }^{32}$ È proprio a partire da questi anni che sull'onda delle urgenze politiche e delle necessità burocratiche e commerciali legate all'espansione della potenza ispanica in Europa si pubblicano un po' in tutti i principali paesi grammatiche di lingua spagnola o comunque strumenti idonei all'apprendimento del castigliano (dizionari, lessici, glossari) da parte degli stranieri. Per un primo sguardo sull'argomento, con numerosi riferimenti bibliografici, cfr. soprattutto J. M. Lope Blanch, Estudios de historia lingüística hispánica, Madrid, Arco/Libros, 1990 (per ciò che
} 
l'autore si mostra consapevole, restino limitati a pochi casi, non può sfuggire la mentalità culturalmente aperta che caratterizza il suo credo linguistico, e soprattutto non può essere ignorata la modernità di una posizione tollerante che, da una parte, tendeva a proteggere sotto la medesima insegna tanto l'espressione scritta quanto quella orale della comunicazione, dall'altra, proponendosi di accogliere senza troppi problemi vocaboli di origine ispanica, dimostrava di percepire la forte unità politico-sociale, che contraddistingueva ormai la nazione spagnola e quella italiana, e al contempo, con un certo anticipo rispetto agli stessi letterati iberici, riconosceva l'assoluta dignità del volgare castigliano e l'importanza da esso destinata ad assumere nel contesto del circuito linguistico e letterario internazionale. A conferma di questa intuizione castiglionesca, non è un caso che solo pochi anni dopo la princeps del trattato (1528), e neanche un paio dopo la pubblicazione della versione castigliana operata da Boscán (1534), Carlo V pronunciasse davanti a papa Paolo III Farnese il celebre discorso in cui sceglieva di esprimersi esclusivamente in «lengua española», nonostante la difficoltà di comprensione degli ambasciatori francesi ${ }^{33}$. I tempi, del resto, erano maturi: Carlo d'Asburgo era ormai padrone di mezza Europa, del Nuovo Mondo e dell'esercito più attrezzato del Cinquecento; inoltre, pochi anni prima era stato incoronato imperatore dalle mani di quello stesso Papa a cui aveva inflitto la vergogna del Sacco. L'inaudita scelta di esprimersi soltanto in castigliano di fronte al seggio di Pietro e ad onta della rappresentanza diplomatica transalpina (scelta compiuta per di più da un monarca non madrelingua e che all'inizio del suo regno non parlava minimamente l'idioma del paese che si accingeva a governare e rendere glorioso), costituiva il primo momento in cui la lingua spagnola acquistava finalmente un prestigio internazionale paragonabile all'estensione dei domini della sua corona. In quell'occasione il motivo politico predominava in assoluto: si trattava in pratica della consapevolezza da parte della Maestà Cesarea dell'affermazione a tutto tondo del potere spagnolo in Europa e nell'orbe conosciuto, al lato del quale, sulla scorta della propaganda nazionalistica di Nebrija, si ribadiva tutto il valore e l'importanza di una «castellana lengua», che ormai risultava davvero essere «en la cumbre» del potere politico, ancor prima che linguistico, internazionale e che pertanto meritava di essere conosciuta - sono parole di Carlo V - «por toda gente christiana».

riguarda l'insegnamento dello spagnolo in Italia, cfr. in part. pp. 27-30); e A. Ramajo Caño, Las gramáticas de la lengua castellana desde Nebrija a Correas, Salamanca, Universidad de Salamanca, 1987.

${ }_{33}$ Sul celebre discorso di Carlo V si sono soffermati molti studiosi, tra cui ricordiamo qui A. Morel Fatio, L'espagnol langue universelle, in Etudes sur l'Espagne, IV serie, Paris, 1925, VI, pp. 189-219; E. Buceta, El juicio de Carlos $V$ acerca del español y otros pareceres sobre las lenguas romances, in «Revista de Filogía española», XXIV, 1937, pp. 11-23;, L. Terracini, Lingua come problema..., cit., in part. pp. 30-31; e, più recentemente e con ulteriori riferimenti, J. Mondejar, Castellano y espanol: dos nombres para una lengua, en su marco literario, ideológico y político, Granada, Universidad de Granada-Editorial Comares, 2002, in part. pp. 6970. 
